\title{
Grading of Non-Alcoholic Fatty Liver Disease on Ultrasound and Its Correlation with Lipid Profile
}

\author{
Sudhir Navale ${ }^{1}$, Dhruv Vala ${ }^{2}$, Madhavi Gupta ${ }^{3}$ \\ ${ }^{1}$ Associate Professor, Department of Radiodiagnosis, Bharati Hospital, ${ }^{2}$ Senior Resident, Departmentof Radiodiagnosis, \\ Bharati Hospital, ${ }^{3}$ Resident, Department of Radiodiagnosis, Bharati Hospital, Bharati Vidyapeeth Deemed University Medical \\ College, Pune, India
}

Corresponding author: Dr Dhruv Vala, Senior Resident, Departmentof Radiodiagnosis, Bharati Hospital, Bharati Vidyapeeth Deemed University Medical College, Pune, India

DOI: http://dx.doi.org/10.21276/ijcmsr.2019.4.3.41

How to cite this article: Sudhir Navale, Dhruv Vala, Madhavi Gupta. Grading of Non-alcoholic fatty liver disease on ultrasound and its correlation with lipid profile. International Journal of Contemporary Medicine Surgery and Radiology. 2019;4(3):C187-C192.

\section{A B S T R A C T}

Introduction: The diagnosis and grading of fatty liver on ultrasonography has been frequently observed these days. The objective of the study was to detect different grades of NAFLD on ultrasonography and correlate it with serum lipid abnormalities.

Material and Methods: This was an observational study on 384 patients referred to Bharati medical college and hospital during June 2018 to May 2019. Patients underwent ultrasonography and were considered "cases" if they had fatty liver. Correlation between NAFLD and serum lipid profile was done.

Results: Of 384 patients, 352 (91.7\%), 30 (7.8\%) and 2 (0.5\%) had grade I, II and III fatty liver respectively. Increased total cholesterol, serum triglycerides, LDL and VLDL were seen in $35.2 \%, 56.8 \%, 34.9 \%$ and $58.9 \%$ of cases respectively; while reduced HDL cholesterol levels in 56.5\%. Total cholesterol to HDL and LDL to HDL ratio was raised in $37 \%$ and $21.1 \%$ of cases respectively. The distribution of mean total cholesterol, triglyceride, LDL levels, total cholesterol to HDL ratio and LDL to HDL ratio differed significantly in our study across three grades of NAFLD. The distribution of abnormality of different components of lipid profile did not differ significantly across fatty liver grades.

Conclusion: Significant positive correlation was noted between NAFLD and increasing levels of serum total cholesterol, triglyceride, LDL, total cholesterol to HDL and LDL to HDL ratios. Significant positive correlation between the fatty liver grades and levels of different components of lipid profile was absent. We can thus conclude that dyslipidemia can be predicted in USG diagnosed NAFLD.

Keyword: NAFLD, Dyslipidemia, USG, Grading, LDL, Triglycerides.

\section{INTRODUCTION}

Fatty liver is one of the commonest problem worldwide. Its prevalence is around $20 \%-30 \%$ in general population in Western countries and 9\% to $32 \%$ in India. ${ }^{1}$ Initially it was termed as a benign pathology but now it is increasingly been recognized as the primary causes of liver-associated morbidities and mortalities. NAFLD spectrum includes simple steatosis and non-alcoholic steatohepatitis (NASH). Studies have demonstrated that non-alcoholic fatty liver disease (NAFLD) can progress to liver cirrhosis, hepatic failure, and further to hepatocellular carcinoma (HCC) as well. ${ }^{2}$ The diagnosis of NAFLD remains underdiagnosed as most of the patients are asymptomatic till terminal stages of disease.

The sensitivity of USG in detecting hepatic steatosis ranges from 60 to $94 \%$ and the specificity from 84 to $95 \% .3,4,5$ Although the biopsy is the gold standard tool for diagnosis of NAFLD, its utility is limited by the invasiveness for just the screening. Fortunately, the presumptive diagnosis can also be made by ultrasound (USG), computed tomography (CT), and magnetic resonance imaging (MRI).

Dyslipidemia is a predisposing factor for complications such as hypertension, atherosclerosis, various heart diseases, renal pathologies, etc. It is possible to correlate ultrasound findings and changes in the dyslipidemia in patients with NAFLD. The objective of the study was to detect different grades of NAFLD on ultrasonography and correlate it with serum lipid abnormalities.

\section{MATERIAL AND METHODS}

This was a prospective observational type of study done at Bharati Vidyapeeth medical college and hospital during June 2016 to May 2018. Total 384 patients were scanned with convex probe and Philip HD 11 machine. The examination was performed with the patient in the supine position. Direct contact scanning technique along with acoustic coupling gel was used. Through scanning was done in all possible approaches. The patient's identity was masked and data used purely for the study purpose. All patients diagnosed as fatty 
liver on USG of the age more than 18 years were included in the study. While the patients with history of regular alcohol intake more than $30 \mathrm{~g} / \mathrm{d}$ in males and more than $20 \mathrm{~g} / \mathrm{d}$ in females were excluded.

Grading of fatty liver was done as -

When the echogenicity was only marginally increased, it was grade I; when the echogenic liver obscured the echogenic walls of portal vein branches, it was grade II, and when the echogenic liver obscured the diaphragmatic outline, it was grade III fatty infiltration ${ }^{6}$.

All the subjects in the study diagnosed as NAFLD on ultrasonography were investigated for serum lipid profile -total cholesterol $(\mathrm{N}<200 \mathrm{mg} / \mathrm{dl})$, HDL $(\mathrm{N}>40 \mathrm{mg} /$ dl for males, $>50$ for females), triglycerides ( $\mathrm{N}<150 \mathrm{mg} /$ $\mathrm{dl})$, LDL $(\mathrm{N}<100 \mathrm{mg} / \mathrm{dl}), \mathrm{VLDL}(\mathrm{N}<12-30 \mathrm{mg} / \mathrm{dl})$ total cholesterol: HDL ratio ( $\mathrm{N}<5$ for male, $<4.5$ for females) and LDL: HDL ratio $(\mathrm{N}<3.6$ for male, $<3.2$ for females). Values outside this range were considered abnormal. Then, a correlation between NAFLD and serum lipid profile was done.

\section{RESULTS}

\section{Age distribution}

Of total 384 subjects included in this study, 34 cases (8.9\%) were between 20.0 - 29.0 years age group, 108 cases $(28.1 \%)$ had their age between 30.0 - 39.0 years, 95 cases $(24.7 \%)$ had their age between $40.0-49.0$ years, 100 cases $(26.0 \%)$ had their age between $50.0-59.0$ years, 36 cases $(9.4 \%)$ had their age between $60.0-69.0$ years, 11 cases $(2.9 \%)$ were between 70.0 - 79.0 years age group (table-1).

So, the mean \pm SD of age of cases studied in the entire study group was $45.5 \pm 11.8$ years. The minimum - maximum range of age was $23-76.0$ years.

\section{Sex distribution}

Of 384 cases studied, 261 cases (68.0\%) were males and 123

\begin{tabular}{|l|c|c|}
\hline Age (Years) & No of cases & Percentage \\
\hline $20-29$ & 34 & 8.9 \\
\hline $30-39$ & 108 & 28.1 \\
\hline $40-49$ & 95 & 24.7 \\
\hline $50-59$ & 100 & 26 \\
\hline $60-69$ & 36 & 9.4 \\
\hline $70-79$ & 11 & 2.9 \\
\hline \multicolumn{2}{|c|}{ Table-1: Age distribution of cases studied $(n=384)}$. \\
\hline
\end{tabular}

\begin{tabular}{|l|c|c|}
\hline & No of cases & Percentage \\
\hline Male & 261 & 68 \\
\hline Female & 123 & 32 \\
\hline \multicolumn{2}{|c|}{ Table-2: Sex distribution of cases studied $(n=384)$} \\
\hline
\end{tabular}

\begin{tabular}{|l|c|c|}
\hline Grade of fatty liver & No of cases & Percentage \\
\hline I & 352 & 91.7 \\
\hline II & 30 & 7.8 \\
\hline III & 2 & 0.5 \\
\hline \multicolumn{2}{|c|}{ Table-3: Distribution of cases studied according to fatty liver } \\
grades on USG ( $n=384)$.
\end{tabular}

cases $(32.0 \%)$ were females. The male to female sex ratio in the entire study group was 2.12: 1.00 (table-2).

Distribution of fatty liver grades on ultrasound

Of total 384 cases, 352 (91.7\%) had Grade I fatty liver, 30 (7.8\%) had Grade II fatty liver and only 2 cases $(0.5 \%)$ had Grade III fatty liver (table-3).

\section{Distribution of grades of fatty liver according to age}

The distribution of grades of fatty liver did not differ significantly across various age groups of cases studied ( $\mathrm{P}$-value $>0.05)$ (table-4).

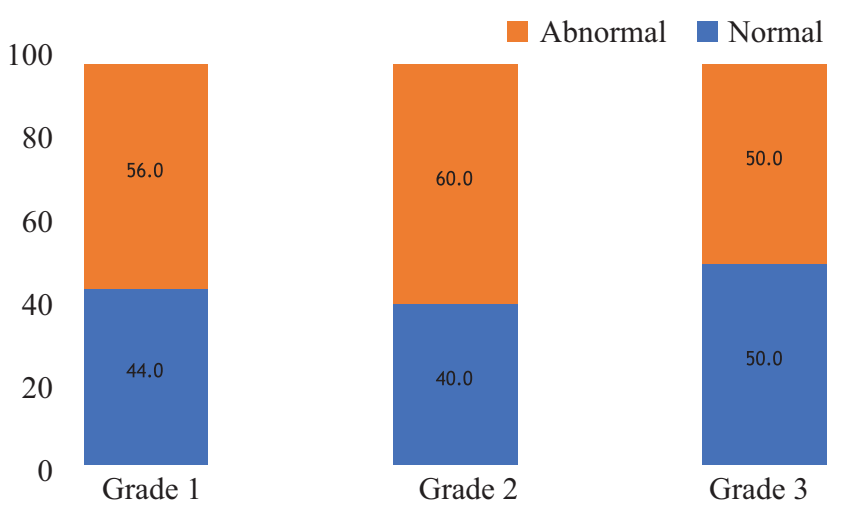

Grades of fatty liver

Figure-1: Distribution of prevalence of abnormality of HDL cholesterol according to the grades of fatty liver $(n=384)$.

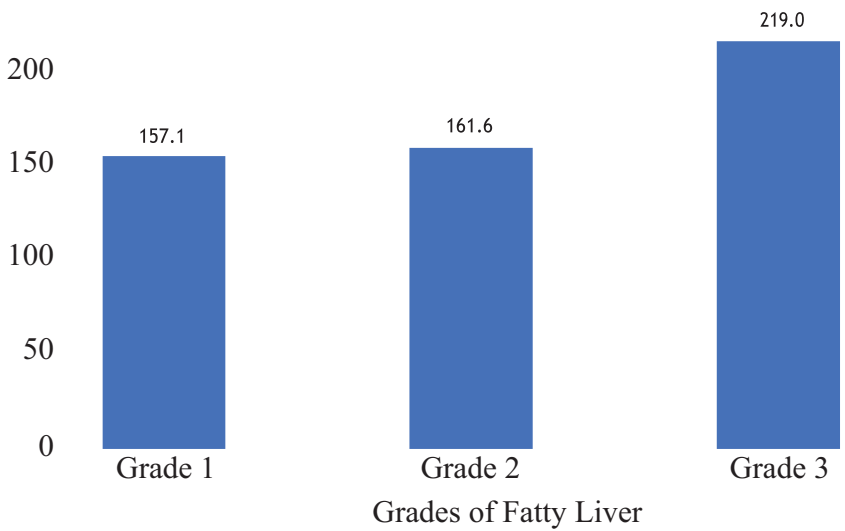

Figure-2: Distribution of mean Triglycerides according to the grades of fatty liver $(n=384)$.
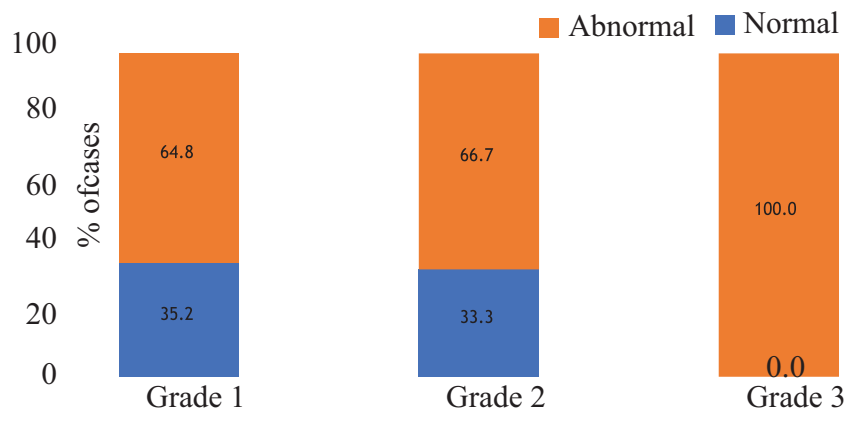

Grades of Fatty Liver

Figure-9(B): Distribution of prevalence of abnormality of LDL cholesterol according to the grades of fatty liver $(n=384)$. 


\begin{tabular}{|c|c|c|c|c|c|c|c|c|}
\hline \multirow[b]{3}{*}{ Age groups (years) } & \multicolumn{6}{|c|}{ Fatty liver (Grades) } & & \\
\hline & \multicolumn{2}{|c|}{ Grade I } & \multicolumn{2}{|c|}{ Grade II } & \multicolumn{2}{|c|}{ Grade III } & \multicolumn{2}{|c|}{ Total } \\
\hline & $\mathrm{n}$ & $\%$ & $\mathrm{n}$ & $\%$ & $\mathbf{n}$ & $\%$ & $\mathrm{n}$ & $\%$ \\
\hline $20-29$ & 32 & 94.1 & 2 & 5.9 & 0 & 0 & 34 & 8.9 \\
\hline $30-39$ & 102 & 94.4 & 6 & 5.6 & 0 & 0 & 108 & 28.1 \\
\hline $40-49$ & 82 & 86.3 & 11 & 11.6 & 2 & 2.1 & 95 & 24.7 \\
\hline $50-59$ & 91 & 91.0 & 9 & 9.0 & 0 & 0 & 100 & 26 \\
\hline $60-69$ & 35 & 97.2 & 1 & 2.8 & 0 & 0 & 36 & 9.4 \\
\hline $70-79$ & 10 & 90.0 & 1 & 9.1 & 0 & 0 & 11 & 2.9 \\
\hline Total & 352 & 91.7 & 30 & 7.8 & 2 & 2.1 & 384 & 100 \\
\hline
\end{tabular}

\begin{tabular}{|l|c|c|c|c|c|}
\hline & \multicolumn{5}{|c|}{ Total cholesterol (mg/dl) } \\
\hline Grades of fatty liver & $\mathbf{N}$ & Mean & S.D. & Minimum & Maximum \\
\hline I & 352 & 188.6 & 39.6 & 73 & 358 \\
\hline II & 30 & 178.9 & 44.0 & 101 & 258 \\
\hline III & 2 & 249.5 & 34.7 & 225 & 274 \\
\hline \multicolumn{2}{|r|}{ Table-5(A): Distribution of mean total cholesterol according to the grades of fatty liver ( $\mathrm{n}=384)}$. \\
\hline
\end{tabular}

\begin{tabular}{|c|c|c|c|c|c|}
\hline \multirow{3}{*}{ Grades of fatty liver } & \multicolumn{5}{|c|}{ Total cholesterol (mg/dl) } \\
\hline & $\mathbf{N}$ & \multicolumn{2}{|c|}{ Normal } & \multicolumn{2}{|c|}{ Abnormal } \\
\hline & 352 & 226 & $64.2 \%$ & 126 & $35.8 \%$ \\
\hline II & 30 & 23 & $76.7 \%$ & 7 & $23.3 \%$ \\
\hline III & 2 & 0 & $0 \%$ & 2 & $100 \%$ \\
\hline
\end{tabular}

\begin{tabular}{|l|c|c|c|c|c|}
\hline & \multicolumn{5}{|c|}{ HDL cholesterol (mg/dl) } \\
\hline Grades of fatty liver & $\mathbf{N}$ & Mean & S.D. & Minimum & Maximum \\
\hline I & 352 & 42.7 & 9.3 & 22 & 72 \\
\hline II & 30 & 40.8 & 9.4 & 28 & 58 \\
\hline III & 2 & 37.7 & 8.1 & 32 & 44 \\
\hline \multicolumn{7}{|c|}{ Table-6(A): Distribution of mean HDL cholesterol according to the grades of fatty liver $(\mathrm{n}=384)}$. \\
\hline
\end{tabular}

\begin{tabular}{|c|c|c|c|c|c|}
\hline \multirow{3}{*}{$\begin{array}{l}\text { Grades of fatty liver } \\
I\end{array}$} & \multicolumn{5}{|c|}{ HDL cholesterol (mg/dl) } \\
\hline & $n$ & \multicolumn{2}{|c|}{ Normal } & \multicolumn{2}{|c|}{ Abnormal } \\
\hline & 352 & 155 & $44 \%$ & 197 & $56 \%$ \\
\hline II & 30 & 12 & $40 \%$ & 18 & $60 \%$ \\
\hline III & 2 & 1 & $50 \%$ & 1 & $50 \%$ \\
\hline
\end{tabular}

\begin{tabular}{|c|c|c|c|c|c|}
\hline \multirow{3}{*}{$\begin{array}{l}\text { Grades of fatty liver } \\
\end{array}$} & \multicolumn{5}{|c|}{ Triglycerides (mg/dl) } \\
\hline & $n$ & \multicolumn{2}{|c|}{ Normal } & \multicolumn{2}{|c|}{ Abnormal } \\
\hline & 352 & 203 & $57.7 \%$ & 149 & $42.3 \%$ \\
\hline II & 30 & 15 & $50 \%$ & 15 & $50 \%$ \\
\hline III & 2 & 0 & $0 \%$ & 2 & $100 \%$ \\
\hline
\end{tabular}

\begin{tabular}{|l|c|c|c|c|c|}
\hline & \multicolumn{5}{|c|}{ LDL cholesterol (mg/dl) } \\
\hline Grades of fatty liver & $\mathbf{n}$ & Mean & S.D. & Minimum & Maximum \\
\hline I & 352 & 114.3 & 33.7 & 11.2 & 247.6 \\
\hline II & 30 & 105.7 & 35.1 & 34.6 & 162.2 \\
\hline III & 2 & 164.5 & 36.1 & 139 & 190 \\
\hline \multicolumn{7}{|r|}{ Table-8(A): Distribution of mean LDL cholesterol according to the grades of fatty liver ( $\mathrm{n}=384)$} \\
\hline
\end{tabular}




\begin{tabular}{|l|c|c|c|c|c|}
\hline Grades of fatty liver & $\mathbf{n}$ & \multicolumn{2}{|c|}{ Normal } & \multicolumn{2}{c|}{ Abnormal } \\
\hline I & 352 & 124 & $35.2 \%$ & 228 & $64.8 \%$ \\
\hline II & 30 & 10 & $33.3 \%$ & 20 & $66.7 \%$ \\
\hline III & 2 & 2 & $0 \%$ & 2 & $100 \%$ \\
\hline \multicolumn{2}{|r|}{ Table-8(B): Distribution of prevalence of abnormality of LDL cholesterol according to the grades of fatty liver ( $\mathrm{n}=384)}$. \\
\hline
\end{tabular}

\begin{tabular}{|l|c|c|c|c|c|}
\hline & \multicolumn{5}{|c|}{ VLDL cholesterol (mg/d) } \\
\hline Grades of fatty liver & $\mathbf{n}$ & Mean & S.D. & Minimum & Maximum \\
\hline I & 352 & 31.3 & 15.8 & 4.0 & 112.2 \\
\hline II & 30 & 32.3 & 16.2 & 9.4 & 72.0 \\
\hline III & 2 & 47.1 & 6.9 & 42.3 & 52.0 \\
\hline \multicolumn{7}{|r|}{} \\
\hline
\end{tabular}

\begin{tabular}{|l|c|c|c|c|c|}
\hline & \multicolumn{5}{|c|}{ Total cholesterol to HDL ratio } \\
\hline Grades of fatty liver & $\mathbf{n}$ & Mean & S.D. & Minimum & Maximum \\
\hline I & 352 & 4.58 & 1.26 & 1.4 & 8.9 \\
\hline II & 30 & 4.59 & 1.46 & 1.8 & 7.4 \\
\hline III & 2 & 6.88 & 2.37 & 5.2 & 8.6 \\
\hline \multicolumn{7}{|c|}{ Table-10(A): Distribution of mean Total cholesterol to HDL ratio according to the grades of fatty liver $(\mathrm{n}=384)}$. \\
\hline
\end{tabular}

\begin{tabular}{|l|c|c|c|c|c|}
\hline & \multicolumn{5}{|c|}{ Total cholesterol to HDL ratio } \\
\hline Grades of fatty liver & $\mathbf{n}$ & \multicolumn{2}{|c|}{ Normal } & 129 & $36.6 \%$ \\
\hline I & 352 & 223 & $63.4 \%$ & 11 & $36.7 \%$ \\
\hline II & 30 & 19 & $63.3 \%$ & 2 & $100 \%$ \\
\hline III & 2 & 0 & $0 \%$ & Abnormal \\
\hline \multicolumn{2}{|l|}{ Table-10(B): Distribution of prevalence of abnormality of Total cholesterol to HDL ratio according to the grades of fatty liver ( $\mathrm{n}=384)}$. \\
\hline
\end{tabular}

\begin{tabular}{|l|c|c|c|c|c|}
\hline & \multicolumn{5}{|c|}{ LDL to HDL ratio } \\
\hline Grades of fatty liver & $\mathbf{n}$ & Mean & S.D. & Minimum & Maximum \\
\hline I & 352 & 2.78 & 0.98 & 0.20 & 6.37 \\
\hline II & 30 & 2.73 & 1.06 & 0.61 & 4.61 \\
\hline III & 2 & 4.55 & 1.91 & 3.2 & 5.9 \\
\hline \multicolumn{7}{|r|}{ Table-11(A): Distribution of mean LDL to HDL ratio according to the grades of fatty liver ( $n=384)}$. \\
\hline
\end{tabular}

\begin{tabular}{|l|c|c|c|c|c|}
\hline & \multicolumn{4}{|c|}{ Total cholesterol to HDL ratio } \\
\hline Grades of fatty liver & $\mathbf{n}$ & 277 & $78.7 \%$ & 75 & $21.3 \%$ \\
\hline I & 352 & 25 & $83.3 \%$ & 5 & $16.7 \%$ \\
\hline II & 30 & 1 & $50.0 \%$ & 1 & $50 \%$ \\
\hline III & 2 & \multicolumn{2}{|c|}{ Normal } & \multicolumn{3}{c|}{ Abnormal } \\
\hline \multicolumn{2}{|r|}{ Table-11(B): Distribution of prevalence of abnormality of LDL to HDL ratio according to the grades of fatty liver ( $\mathrm{n}=384)}$. \\
\hline
\end{tabular}

Distribution of mean total cholesterol according to grades of fatty liver

The distribution of mean total cholesterol levels differs significantly across three grades of fatty liver $(\mathrm{P}$-value $<0.05)$ (table-5a).

Distribution of abnormality of total cholesterol according to grades of fatty liver

The distribution of prevalence of abnormality of total cholesterol levels did not differ significantly across three grades of fatty liver ( $\mathrm{P}$-value $>0.05)$ (table-5b).

Distribution of mean HDL cholesterol according to the grades of fatty liver

The distribution of mean HDL cholesterol did not differ significantly across three grades of fatty liver ( $\mathrm{P}$-value $>0.05)$ (table-6a).

Distribution of prevalence of abnormality of HDL cholesterol according to the grades of fatty liver The distribution of prevalence of abnormality of HDL cholesterol levels did not differ significantly across three grades of fatty liver (P-value $>0.05)$ (table-6b)

Distribution of mean Triglycerides according to the grades of fatty liver

The distribution of mean Triglycerides did not differ significantly across three grades of fatty liver ( $\mathrm{P}$-value $>0.05)$ (fig-1). 
Distribution of prevalence of abnormality of Triglycerides according to the grades of fatty liver

The distribution of prevalence of abnormality of Triglyceride levels did not differ significantly across three grades of fatty liver ( $\mathrm{P}$-value $>0.05)$ (fig-2), (table-7b).

\section{Distribution of mean LDL cholesterol according to the grades of fatty liver}

The distribution of mean LDL cholesterol differs significantly across three grades of fatty liver $(\mathrm{P}$-value $<0.05)($ table-8a).

Distribution of prevalence of abnormality of LDL cholesterol according to the grades of fatty liver:

The distribution of prevalence of abnormality of LDL cholesterol levels did not differ significantly across three grades of fatty liver ( $\mathrm{P}$-value $>0.05$ ) (table-8b).

Distribution of mean VLDL cholesterol according to the grades of fatty liver

The distribution of mean VLDL cholesterol did not differ significantly across three grades of fatty liver $(\mathrm{P}$-value $>0.05)$ (table-9a).

Distribution of prevalence of abnormality of VLDL cholesterol according to the grades of fatty liver

The distribution of prevalence of abnormality of VLDL cholesterol levels did not differ significantly across three grades of fatty liver (P-value $>0.05)$ (fig-3).

\section{Distribution of mean Total cholesterol to HDL ratio according to the grades of fatty liver}

The distribution of mean total cholesterol to HDL ratio differs significantly across three grades of fatty liver $(\mathrm{P}$-value $<0.05)($ fig-4).

\section{Distribution of prevalence of abnormality of Total} cholesterol to HDL ratio according to the grades of fatty liver

The distribution of prevalence of abnormality of Total cholesterol to HDL ratio did not differ significantly across three grades of fatty liver (P-value $>0.05)$ (table-10b).

Distribution of mean LDL to HDL ratio according to the grades of fatty liver

The distribution of mean LDL to HDL ratio differs significantly across three grades of fatty liver $(\mathrm{P}$-value $<0.05)$ (table-11a).

\section{Distribution of prevalence of abnormality of LDL to HDL ratio according to the grades of fatty liver}

The distribution of prevalence of abnormality of LDL to HDL ratio did not differ significantly across three grades of fatty liver (P-value >0.05) (table-11b).

\section{DISCUSSION}

In our study mean $\pm \mathrm{SD}$ of age of cases studied in the entire study group was $45.5 \pm 11.8$ years. The minimum - maximum range of age was $23-76.0$ years. Most of the western studies have reported the mean age of NAFLD between 41-45 years. In Indian studies mean age was reported to be $(42.90+10.54)$ years by Roli Agarwal et $\mathrm{al}^{7} 55.4$ years by Amarapurkar et al80, $(49.14+9.65)$ years by Mahaling et al ${ }^{10}$
So, age distribution in our study was comparable to other studies for international and Indian population.

Among the 384 patients included in the study, 352 (91.7\%), $30(7.8 \%)$ and $2(0.5 \%)$ had grade I, II and III fatty liver respectively. This is similar to $83 \%, 17 \%$ and $0 \%$ for grade I, II and III respectively in a study by Bhusal et al. ${ }^{8}$ Similar distribution was seen in study by Sen et al. ${ }^{9}$ However, studies by Mahaling et $\mathrm{al}^{10}$ and Roli Agarwal et $\mathrm{al}^{7}$ had higher percentages of grade II and III cases.

In our study, distribution of cases was studied according to age and fatty liver grades on USG by Chi-Square test. It was found statistically insignificant $(\mathrm{P}>0.05)$ Thus the 61 distribution of grades of fatty liver did not differ significantly across various age groups of cases studied.

In our study raised total cholesterol, serum triglycerides, LDL and VLDL were seen in $35.2 \%, 56.8 \%, 34.9 \%$ and $58.9 \%$. Raised total cholesterol, serum triglycerides, LDL and VLDL were seen in $45.71 \%, 67.14 \%, 34.28 \%$ and $25.71 \%$ of cases respectively by Mahaling et al. ${ }^{10}$ Roli Agrawal et $\mathrm{al}^{7}$ reported hypercholesterolemia in $21.8 \%$ patients, hypertriglyceridemia in $63.7 \%$, elevated LDL in $25 \%$ of patients and elevated VLDL in $56.5 \%$ of patients. Bhusal et $\mathrm{al}^{8}$ reported total cholesterol, serum triglycerides, LDL, were raised in 53\%, 59\%, and $72 \%$ respectively.

In our study decrease in HDL cholesterol was seen in $56.5 \%$. Mahaling et $\mathrm{al}^{10}$, Roli Agrawal et $\mathrm{al}^{7}$ and Bhusal et $\mathrm{al}^{8}$ reported low HDL in $62.8 \%, 45.1 \%$ and $57 \%$ respectively. In addition to these, total cholesterol to HDL ratio and LDL to HDL ratio was raised in $37 \%$ of cases and $21.1 \%$ of cases respectively. Data for these ratios was not available in similar studies for comparison. The distribution of mean total cholesterol, triglyceride, LDL levels, total cholesterol to HDL and LDL to HDL ratios differs significantly in our study (P0.05) across three grades of fatty liver using the same test. In Mahaling et al study ${ }^{10}$, serum total cholesterol, serum HDL, serum LDL and VLDL showed statistical significance with increasing grades of NAFLD. Serum triglyceride showed no statistical significance with increasing grades of NAFLD. In Bhusal et al ${ }^{8}$ study there was significant positive correlation of presence of NAFLD with increasing levels of serum total cholesterol, LDL and triglyceride and significantly decreasing HDL.

Distribution of prevalence of abnormality of different components of lipid profile did not differ significantly across three grades of fatty liver. Similar results were found by Bhusal et $\mathrm{al}^{8}$ Therefore, Ultrasonography is a cheap, fast, and widely available imaging technique for applications in fatty liver. US has been reported to have a sensitivity ranging from $60 \%$ to $94 \%$ and a specificity of $66 \%$ to $95 \% 75$; lower sensitivities are frequently observed in patients with mild disease. ${ }^{11}$ Limitations of US include it being subject to interobserver variability, difficulty in obese patients, and the high proportion of NAFLD patients with coexisting obesity.

\section{CONCLUSION}

Study thus concluded that the fatty liver was more common in male sex with the commonest age group affected being of 20-30 years. Almost three quarters of fatty liver patients had normal liver size while one quarter had increased size of 
the liver. Majority of patient with NAFLD had dyslipidemia. There was significant positive correlation of presence of NAFLD with increasing levels of mean serum total cholesterol, triglyceride, LDL levels, total cholesterol to HDL and LDL to HDL ratios. There was no significant positive correlation of presence of NAFLD with increasing level of mean serum VLDL and decreasing level of mean serum HDL. Ultrasound is a $1^{\text {st }}$ line imaging tool to diagnose and subsequently grade the steatosis. It is cheap, widely available. However, it has drawbacks such as observer dependency. From the study, it was shown that increasing grades of fatty liver had significant association with deranged lipid profile. Deranged lipid profile is associated with cardiovascular problems. Hence, increasing grades of fatty liver has indirect relationship with cardiovascular problems.

\section{REFERENCE}

1. Duseja A. Nonalcoholic fatty liver disease in India-a lot done, yet more required!. Indian Journal of Gastroenterology. 2010;29(6):217-25

2. Mahaling DU, Basavaraj MM, Bika AJ. Comparison of lipid profile in different grades of non-alcoholic fatty liver disease diagnosed on ultrasound. Asian Pacific journal of tropical biomedicine. 2013;3(11):907-12.

3. Joseph AE, Saverymuttu SH, Al-Sam S, Cook MG, Maxwell JD. Comparison of liver histology with ultrasonography in assessing diffuse parenchymal liver disease. Clinical radiology. 1991;43(1):26-31

4. Debongnie JC, Pauls C, Fievez M, Wibin E. Prospective evaluation of the diagnostic accuracy of liver ultrasonography. Gut. 1981;22(2):130-5.

5. Saverymuttu SH, Joseph AE, Maxwell JD. Ultrasound scanning in the detection of hepatic fibrosis and steatosis. Br Med J (Clin Res Ed). 1986;292(6512):13-5.

6. Saadeh S, Younossi ZM, Remer EM, Gramlich T, Ong JP, Hurley M, Mullen KD, Cooper JN, Sheridan MJ. The utility of radiological imaging in nonalcoholic fatty liver disease. Gastroenterology. 2002;123(3):745-50

7. Agrawal R, Mishra S, Dixit VK, Rai S. Association of non-alcoholic fatty liver disorder with obesity. Indian J Prev Soc Med. 2009;40:126-9.

8. Bhusal KR, Simkhada R, Nepal P. Lipid profile in different grades of Ultrasonic NonAlcoholic Fatty Liver Disease. Journal of College of Medical Sciences-Nepal. 2017;13(2):258-61.

9. Sen A, Kumar J, Misra RP, Uddin M, Shukla PC. Lipid profile of patients having nonalcoholic fatty liver disease as per ultrasound findings in north Indian population: A retrospective observational study. Journal of Medical \& Allied Sciences. 2013;3(2):59.

10. Mahaling DU, Basavaraj MM, Bika AJ. Comparison of lipid profile in different grades of non-alcoholic fatty liver disease diagnosed on ultrasound. Asian Pacific journal of tropical biomedicine. 2013;3(11):907-12.

11. Schwenzer NF, Springer F, Schraml C, Stefan N, Machann J, Schick F. Non-invasive assessment and quantification of liver steatosis by ultrasound, computed tomography and magnetic resonance. Journal of hepatology. 2009;51(3):433-45.

\section{Source of Support: Nil; Conflict of Interest: None}

Submitted: 13-07-2019; Accepted: 17-08-2019; Published online: 15-09-2019 